Exosomes, small (30-150nm) extracellular vesicles of endocytic origin, are present in all body fluids of cancer patients. Tumor-derived exosomes, TEX, emerge as potentially promising non-invasive biomarkers of tumor progression and of immune cell dys function in cancer. Exosomes isolated from plasma by size exclusion chromatography can be fractionated into TEX and non-TEX by immune capture on beads. Profiling of molecular and genetic contents of TEX shows that levels if immunosuppressive proteins, such as PD-L1, carried by TEX associate with disease progression. The data suggest that TEX have a to serve as tumor surrogates, while immune cell-derived exosomes might serve as biomarkers of immune dysfunction in cancer.

Key words: exosomes, tumor-derived exosomes (TEX), cancer prognosis, cancer immunotherapy.

Contemp Oncol (Pozn) 2018; 22 (1A): 38-40 DOI: https://doi.org/10.5114/wo.2018.73882

\section{The emerging role of plasma exosomes in diagnosis, prognosis and therapies of patients with cancer}

\author{
Theresa L. Whiteside
}

University of Pittsburgh Cancer Institute, Hillman Cancer Center, Pittsburgh, USA

Exosomes are currently emerging as promising noninvasive correlates of tumor progression and as markers of immune evasion of cancer. Exosomes are the smallest of extracellular vesicles (EVs) produced by all cells. They are virus size (30-150 nm), membrane-bound vesicles that originate from the endocytic compartment of the parent cell and carry endosomal markers such as ALIX or TSG101. Because of their endosomal origin, exosomes are distinct from larger EVs, also called microvesicles (MV), which are formed by "pinching off" the cellular membrane and from the even larger apoptotic bodies. Today, the nomenclature of EVs is not entirely established, and there is a possibility that an overlap exists among various EVs. Tumor cells produce large quantities of exosomes, which are called tumor-derived exosomes (TEX) and which are of special interest, as their molecular and genetic contents in part resemble those of the parent cell. Thus, TEX are considered to be similar to tumor cells from which they originate and, because TEX circulate freely in the body fluids, they are thought of as potential "liquid tumor biopsies". This concept, while attractive from the cancer biomarker prospective, has yet to be validated. TEX carry a rich membrane-associated cargo of immunosuppressive and immunostimulatory proteins, MHC class I and II antigens, lipids, and glycolipids. In their lumen, TEX have a large portfolio of cellular proteins, enzymes, soluble factors and nucleic acids, including miRNAs [1-3].

Exosomes present in plasma can be isolated by many different methods [4]. The method of choice used in my laboratory is size exclusion chromatography (SEC) on small Sepharose-packed columns, allowing for the isolation of morphologically intact, non-aggregated, partly free of plasma proteins and functionally active exosomes in the void volume (fraction \#4) as described [5]. Total exosome fractions isolated by this mini-SEC method from plasma of cancer patients contain TEX and non-tumor-derived exosomes in various ratios, depending on the individual patient. The protein content of exosomes isolated from plasma is informative and, as reported previously, might predict survival of patients with melanoma [6]. The protein content of exosomes in cancer patients' plasma (in $\mu g$ protein $/ \mathrm{ml}$ plasma) is significantly elevated relative to that in NC plasma [6-8]. Further, the total exosome protein content is significantly higher in the plasma of cancer patients with late vs early stage disease and decreases in response to oncologic therapies [5, 8]. Thus, total protein content of plasma-derived exosomes appears to be a useful predictor of disease activity, stage, lymph node involvement, response to therapy and overall survival in patients with solid or hematological malignancies.

The cargo of isolated total plasma exosomes can be examined by various procedures, including mass spectrometry [8-10]. However, some of the exosome cargo components, especially biologically significant ligands or soluble factors, are present in femtomolar quantities, so that their detection 
by, e.g., mass spectrometry, is not always successful, and amplification with cargo-specific antibodies may be necessary to confirm their presence and levels in exosomes. Western blots are often used for studies of exosome cargo [8], and our recent results suggest that semi-quantitative densitometry analysis of protein bands (integrated pixel value $=$ image intensity $x$ area of the band) is informative with respect to disease activity or progression [8]. Specifically, densitometry analysis of exosome Western blots for immunoregulatory proteins, such as PD-L1 or TGF- $\beta$ / LAP, demonstrated that the levels of these proteins in total plasma exosomes were significantly associated with disease activity, stage or grade [8].

A better quantitative analysis of the exosome cargo can be obtained using flow cytometry. A method for capture of exosomes on streptavidin-coated magnetic beads was developed that allows for capture of $\mathrm{CD} 3^{+}$exosomes using biotin-labeled anti-CD63 Ab starting with fraction \#4 of exosomes isolated by mini-SEC (M-N Theodoraki \& T.L. Whiteside, unpublished data). The $\mathrm{CD}^{+} 3^{+}$exosomes captured by the beads are detected with a fluorescent antibody specific for a designated exosome cargo component by flow cytometry. This method was used for quantitation of individual cargo components, such as PD-1 or PD-L1. We showed that the levels of PD-1 or PD-L1 present on exosomes derived from plasma of patients with head and neck cancer (HNC) significantly correlated with clinicopathological factors such as disease activity, stage and tumor grade. Thus, PD-1 and especially PD-L1 levels in exosomes inform about the disease progression, confirming that exosomes have the potential to serve as disease biomarkers.

PD-L1 is a ligand able to induce immune suppression in activated T cells which express PD-1. When PD-L1 carrying exosomes with high or low levels of this protein were co-incubated with human activated CD3+CD69+ T lymphocytes, down-regulation of CD69 expression was significantly inhibited. This inhibition was almost completely blocked by anti-PD1 Ab. Similarly, when plasma-derived exosomes were co-incubated with activated CD8+ T cells, rapid apoptosis of T cells was observed [8]. These data indicated that plasma-derived exosomes or TEX modulate immune cell activity and that exosomes have the potential to also serve as immune biomarkers.

Plasma-derived exosomes in patients with cancer are mixtures of tumor-derived vs. normal cell-derived vesicles. Therefore, to determine which of the two subsets is responsible for immune modulation, it is necessary to separate them, starting with total plasma exosomes in fraction \#4. We use immune capture with magnetic beads and Abs specific for an epitope present exclusively on tumor but not normal cells for this purpose. We have successfully isolated TEX from non-TEX using exosomes isolated from plasma of melanoma patients. Studies are currently in progress to determine which of the two exosome subsets modulates immune suppression. These studies are expected to confirm our earlier results obtained with exosomes produced by melanoma cell lines that TEX carry immunosuppressive cargos and thus could contribute to tumor immune escape $[11,12]$.
More recently, we showed that exosomes in plasma of cancer patients with advanced malignancies can interfere with immunotherapy [13]. Plasma-derived exosomes from patients with recurrent/refractory AML were isolated prior to adoptive therapy with therapeutic NK92 cells (Neukoplast). NK92 cells were delivered to patients enrolled in a phase I clinical trial. These exosomes carried numerous immunoinhibitory proteins (PD-L1, FasL, CD39/CD73, COX2) in addition to several leukemia-associated antigens (LAA). These exosomes induced massive down-regulation in functions of NK-92 cells used for therapy. Brief co-incubation of these exosomes with NK92 cells led to a significant loss of NK-92 expression, decrease in cytotoxicity against leukemic blasts or K562 targets, and in NK-92 cell migration toward leukemia cells in transwell experiments. In aggregate, our data suggest that NK-92 cells, which do not internalize PKH26-labeled exosomes even during prolonged co-incubation, receive multiple inhibitory signals via cognate cell surface receptors that result in simultaneous silencing of several functional pathways.

Overall, our studies of plasma-derived exosomes in patients with malignancies indicate that TEX could serve as tumor "surrogates," reflecting in part the molecular and genetic content of the parent tumor cells (i.e., as a "liquid biopsy"). In addition, immune cell-derived exosomes (IEX) carry a molecular cargo derived from immune cells and might serve as biomarkers of immune dysfunction which facilitates tumor escape. The fractionation of plasma-derived exosomes into TEX and IEX is expected to identify biomarkers relevant to the tumor as well as immune competence of the cancer patient.

This work was supported in part by NIH grants RO1 CA168628 and R21 CA205644 to TLW.

The author declares no conflicts of interest.

\section{References}

1. Abels ER, Breakefield XO. Introduction to Extracellular Vesicles: Biogenesis, RNA Cargo Selection, Content, Release, and Uptake. Cell Mol Neurobiol 2016; 36: 301-12.

2. Raposo G, Stoorvogel W. Extracellular vesicles: exosomes, microvesicles, and friends. J Cell Biol 2013; 200: 373-83.

3. Cocucci E, Meldolesi J. Ectosomes and exosomes: shedding the confusion between extracellular vesicles. Trends Cell Biol 2015; 25 : 364-72.

4. Taylor DD, Shah S. Methods of isolating extracellular vesicles impact down-stream analyses of their cargoes. Methods 2015; 87: 3-10.

5. Hong CS, Funk S, Muller L et al. Isolation of biologically active and morphologically intact exosomes from plasma of patients with cancer. J Extracell Vesicles 2016; 5: 29289.

6. Peinado H, Aleckovic M, Lavotshkin S, et al. Melanoma exosomes educate bone marrow progenitor cells toward a pro-metastatic phenotype through MET. Nat Med 2012; 18: 883-91.

7. Hong CS, Muller L, Boyiadzis M, Whiteside TL. Isolation and characterization of CD34+ blast-derived exosomes in acute myeloid leukemia. PLoS One 2014; 9: e103310.

8. Funk S, Floros T, Hong CS, et al. Suppression of lymphocyte functions by plasma exosomes correlates with disease activity in patients with head and neck cancer. Clin Cancer Res 2017; In press. 
9. Melo SA, Luecke LB, Kahlert C et al. Glypican-1 identifies cancer exosomes and detects early pancreatic cancer. Nature 2015; 523: 177-82.

10. Kowal J, Arras G, Colombo M et al. Proteomic comparison defines novel markers to characterize heterogeneous populations of extracellular vesicle subtypes. Proc Natl Acad Sci U S A 2016; 113: E968-77.

11. Wieckowski EU, Visus C, Szajnik M et al. Tumor-derived microves icles promote regulatory $T$ cell expansion and induce apoptosis in tumor-reactive activated CD8+ T lymphocytes. J Immunol 2009; 183: 3720-30.

12. Whiteside TL. Exosomes and tumor-mediated immune suppres sion. J Clin Invest 2016; 126: 1216-23.

13. Hong CS, Sharma P, Yerneni SS, Simms P, Jackson EK, Whiteside TL, Boyiadzis M. Circulating exosomes carrying an immunosuppressive cargo interfere with cellular immunotherapy in acute myeloid leukemia. Sci Rep 2017; 7: 14684.

\section{Address for correspondence}

\section{Theresa L. Whiteside}

University of Pittsburgh Cancer Institute

Hillman Cancer Center

5117 Centre Avenue, Suite 1.27

Pittsburgh, PA 15213, USA

tel. (412) 624-0096

fax (412) 624-0264

email:whitesidetl@upmc.edu 